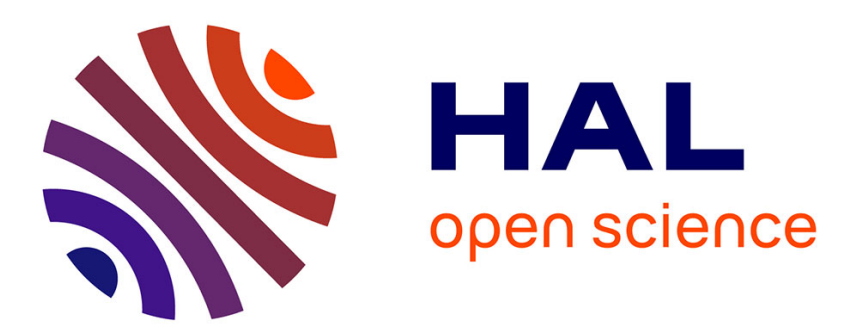

\title{
Neuroanatomical correlates of haptic object processing: combined evidence from tractography and functional neuroimaging
}

Haemy Lee Masson, Hyeok-Mook Kang, Laurent Petit, Christian Wallraven

\section{- To cite this version:}

Haemy Lee Masson, Hyeok-Mook Kang, Laurent Petit, Christian Wallraven. Neuroanatomical correlates of haptic object processing: combined evidence from tractography and functional neuroimaging. Brain Structure and Function, 2018, 223 (2), pp.619 - 633. 10.1007/s00429-017-1510-3 . hal01773704

\author{
HAL Id: hal-01773704 \\ https://hal.science/hal-01773704
}

Submitted on 5 Feb 2021

HAL is a multi-disciplinary open access archive for the deposit and dissemination of scientific research documents, whether they are published or not. The documents may come from teaching and research institutions in France or abroad, or from public or private research centers.
L'archive ouverte pluridisciplinaire HAL, est destinée au dépôt et à la diffusion de documents scientifiques de niveau recherche, publiés ou non, émanant des établissements d'enseignement et de recherche français ou étrangers, des laboratoires publics ou privés. 


\section{Neuroanatomical correlates of haptic object processing: combined evidence from tractography and functional neuroimaging}

Lee Masson, Haemy ${ }^{1 *} \&$ Kang, Hyeok-mook ${ }^{2} \&$ Petit, Laurent $^{3} \&$ Wallraven, Christian ${ }^{2 *}$

${ }^{1}$ Department of Brain and Cognition, KU Leuven, 3000 Leuven, Belgium

${ }^{2}$ Department of Brain and Cognitive Engineering, Korea University, Seoul 136-713, Korea

${ }^{3}$ Groupe d'Imagerie Neurofonctionnelle, Institut Des Maladies Neurodégénératives - UMR 5293,

CNRS, CEA University of Bordeaux, Bordeaux, France

*Contributed equally to this article.

Corresponding author: Christian Wallraven, wallraven@,korea.ac.kr, (+82)1050345925

This research was supported by the Basic Science Research Program through the National Research Foundation of Korea (NRF) funded by the Ministry of Science, ICT \& Future planning (NRF2015S1A5A8018) and the Brain Korea 21plus program through the National Research Foundation of Korea (NRF) funded by the Ministry of Education. 


\section{Abstract}

Touch delivers a wealth of information already from birth, helping infants to acquire knowledge about a variety of important object properties using their hands. Despite the fact that we are touch experts as much as we are visual experts, surprisingly little is known how our perceptual ability in touch is linked to either functional or structural aspects of the brain. The present study therefore investigates and identifies neuroanatomical correlates of haptic perceptual performance using a novel, multi-modal approach. For this, participants' performance in a difficult shape categorization task was first measured in the haptic domain. Using a multi-modal functional magnetic resonance imaging - diffusion-weighted magnetic resonance imaging analysis pipeline, functionally-defined and anatomically-constrained white-matter pathways were extracted and their microstructural characteristics correlated with individual variability in haptic categorization performance. Controlling for the effects of age, total intracranial volume and head movements in the regression model, haptic performance was found to correlate significantly with higher axial diffusivity $(A D)$ in functionally-defined superior longitudinal fasciculus (fSLF) linking frontal and parietal areas. These results were further localized in specific subparts of fSLFs. Using additional data from a second group of participants, who first learned the categories in the visual domain and then transferred to the haptic domain, haptic performance correlates were obtained in the functionally-defined inferior longitudinal fasciculus (fILF). Our results implicate SLF as an important white-matter track in processing touch-specific information during object processing whereas ILF relays visually-learned information during haptic processing. Taken together, the present results chart for the first time potential neuroanatomical correlates and interactions of touch-related object processing.

Key words: DWI; fMRI; multisensory processing; object shape; superior longitudinal fasciculus; inferior longitudinal fasciculus 


\section{Introduction}

The human brain is able to effortlessly recognize and categorize objects from visual input despite the enormous variability in object colours, shapes, and materials that exist in the world. What is often forgotten in the context of these impressive visual processing capabilities we possess, however, is that much of this knowledge has been acquired in a multisensory setting: from day one, infants interact with the world and its objects using all sensory modalities, including vision, audition, smell, taste, and - for the purposes of this paper - touch. The sense of touch provides the infant with a wealth of input about a variety of object properties, such as shape, body-relative size, texture, weight, elasticity, and temperature, all of which can be readily integrated with the visual input the brain receives during manipulation of objects. Hence, touch in this context serves as a bootstrapping modality without which vision would not be able to acquire its extraordinary expertise in object processing. Indeed, previous studies provided evidence that shape information obtained solely through the touch modality is as reliable and robust as that obtained through vision (Klatzky, et al., 1985), and that touch can accurately reconstruct highly abstract and complex physical shape spaces (Gaißert, et al., 2010; Lee Masson, et al., 2016a).

When looking into evidence from functional neuroimaging studies that illustrates how haptic shape processing may be represented in the brain, several studies have uncovered a variety of cortical areas, including primary somatosensory cortices (SI), the parietal lobe for haptic shape information (Bodegard, et al., 2001; Reed and Caselli, 1994), as well as the lateral occipital cortex (LOC) as a potential multisensory convergence area (Amedi, et al., 2002; James, et al., 2007; Lacey, et al., 2009; Lee Masson, et al., 2016a).

When it comes to neuroanatomical evidence, however, only one prior study to our knowledge has addressed which white matter (WM) pathways may relay haptic shape information. In this previous study of ours, we found WM pathways that were able to reflect individual variability in cross-modal (visual to haptic) categorization performance - in particular, we found correlations of cross-modal performance with micro-structural properties of the inferior longitudinal fasciculus (ILF) and with the superior longitudinal fasciculus linking frontal and temporal cortices while traversing the parietal lobe (SLF $\mathrm{ft}_{\mathrm{t}}$ (Lee Masson, et al., 2016b). The aim of that study, however, was to look for neuroanatomical correlates of information that is recognized haptically, but that had been learned visually - hence, these 
results may be influenced, for example, by effects of visual imagery. Indeed, it has been shown that neural representation of haptic shape properties can be modulated by prior visual knowledge (Lee Masson, et al., 2016a), leaving the question open whether the individual variance in the microstructural properties of white matter pathways would mediate haptic shape processing even in the absence of visual knowledge.

Therefore, the aim of the present study is, first, to extract functionally-defined and anatomicallyconstrained WM pathways as track of interests (fILF and fSLFs) based on the cortical regions activated during either visual or haptic shape recognition task by applying combined in vivo DWI and fMRI data. Second, we examine whether the individual microstructural characteristics of these functionallydefined WM pathways can predict haptic performance differences during a difficult categorization task without prior visual knowledge. Third, we compare the results obtained in the present study to those from re-analyzed data of our previous work (Lee Masson, et al., 2016b) in order to better characterize the neuroanatomical correlates of touch-related processing.

In order to achieve these goals, we implement a behavioral task closely modeled after our previous studies (Lee Masson, et al., 2016b; Wallraven, et al., 2014). In this task, participants learn to categorize novel, complex three-dimensional shapes using the haptic modality. The dependent variable is learning speed and measures how many training blocks are required to learn the categories. Similarly to our previous study, we are here interested in determining individual variance in the haptic shape categorization performance rather than to simply look at "average performance". Subsequently, participants are scanned using DWI protocols, and microstructural characteristics of major functionally-defined WM bundles are extracted and correlated with haptic performance (that is, learning speed).

Previous evidence has found that ILF can be viewed as a higher-order, visually-associative WM tract involved, for example, in face processing (Coello, et al., 2013; Grossi, et al., 2014), scene understanding (Tavor, et al., 2014), object processing (Ortibus, et al., 2012), visual memory (Shinoura, et al., 2007), and digit-symbol coding (Lee Masson, et al., 2016b). We therefore hypothesize that individual variance in haptic performance would not correlate with microstructural properties of the fILF as visual knowledge about the novel objects is no longer available during the haptic shape categorization task. In contrast, given the location of fSLFs that traverse motor and somatosensory 
areas, the fSLFs may relay haptic shape information regardless of the availability of visual processing, such as visual imagery. Indeed, SLFs were proven to be WM pathways related with the tasks requiring sensory-motor interaction (Vry, et al., 2015).

In sum, with our multi-modal approach, we hypothesize that functionally-defined WM tracks extracted from the evoked neural activation during visual object recognition task may be able to reflect visuohaptic performance while WM tracks extracted by delineating the neural activity during haptic recognition task may express haptic performance regardless of prior visual knowledge.

\section{Materials and methods}

\section{Participants}

Thirty-nine right-handed healthy adults (age 17-29; mean age $=21.8$; standard deviation $=2.5 ; 22$ women) without history of neurological deficits or haptic perceptual impairments participated in this study that received prior approval by the Korea University Institutional Review Board (1040548-KUIRB-14-133-A-1). All participants were provided with written informed consent.

\section{Stimuli}

The stimuli consisted of 10 novel three-dimensional objects defined by a one-dimensional continuous stimulus space. Among these, the 2 stimuli at either ends of the continuum were selected to represent either category A or B as "prototypical exemplars". The other eight stimuli were used for the shape categorization training (Lee Masson, et al., 2016b; Wallraven, et al., 2014). Stimuli were printed out as tangible objects (average measurements: $7 \times 7 \times 7 \mathrm{~cm}$, average weight: $97.88 \mathrm{~g}$ ) using a 3D printer (Zprinter650, 3DSystems, USA).

\section{Behavioral experiments}

Participants were trained to correctly categorize the object shape by touch. During the experiment, the participant and the experimenter were seated facing each other at a table. A black curtain was installed in the middle of the table and the participant was blindfolded so as to block visual input. The participant was instructed to reach out the right hand through the curtain to explore an object fixed to a stand. An experimenter presented the stimuli at random angles while following the auditory cues for the presentation timing through a headphone and also recorded the verbal responses of the participant. 
Throughout the experiment, care was taken that the participant did not receive visual input about the objects.

The training started with presentation of the two, verbally labeled prototype exemplars for 10 seconds each to provide anchoring information for the categories. Eight objects from the training stimuli set were presented in a random order for $6 \mathrm{~s}$ during each following trial (see Lee Masson, 2016b). These 10 trials (two exemplars $(10 \mathrm{~s})+$ eight objects $(6 \mathrm{~s})$ ) composed one block of training. During the training, the participant reported if the object belonged to either category A or B, followed by verbal feedback provided by the experimenter whether the response was correct or not. According to our criterion, training was continued until participants correctly categorized all 8 exemplars in two consecutive blocks. In case the participant could not reach the criterion even after 20 blocks, the training was stopped since further training could be confounded by the degree of fatigue caused by excessive touch exploration and blindfolding.

\section{MRI acquisition}

MRI data were acquired a few days after the behavioral experiments on a SIEMENS Trio 3T scanner (Siemens Medical Systems, Erlangen, Germany) with a 32-channel SENSE head-coil (Brain-imagingcenter, Korea University, Seoul, South Korea). T1-weighted sagittal high-resolution MPRAGE sequence provided individual structural anatomical images (repeat time $(\mathrm{TR})=2250 \mathrm{~ms}$, echo time $(\mathrm{TE})=3.65 \mathrm{~ms}$, flip angle $(\mathrm{FA})=9^{\circ}$, voxel size $=1 \times 1 \times 1 \mathrm{~mm}, 192$ axial slices) while diffusion weighted spin-echo echo-planar-imaging (DWI-EPI) pulse sequence provided whole brain diffusion-weighted images $((\mathrm{TR})=6400 \mathrm{~ms}$, echo time $(\mathrm{TE})=84 \mathrm{~ms}, \mathrm{~b}=1000 \mathrm{~s} / \mathrm{mm} 2$, with 64 diffusion gradient orientations in addition to one non-weighted b0 image, 52 slices, voxel size $=2.2 \times 2.2 \times 2 \mathrm{~mm}$ ).

To achieve functionally defined white matter tracks, functional images were obtained with a gapless, echo planar imaging sequence $\left(\mathrm{TR}=3000 \mathrm{~ms}, \mathrm{TE}=30 \mathrm{~ms}, \mathrm{FA}=60^{\circ}, \mathrm{FOV}=250 \times 250 \mathrm{~mm}\right.$, in-plane matrix $=128 \times 128$, voxel size $=2 \times 2 \times 4 \mathrm{~mm}, 30$ slices). Dummy scans were performed during the first $9 \mathrm{~s}$ for steady-state magnetization.

Image processing and extraction of the tracks of interest (TOIs) from whole brain tractogram 
As a preprocess procedure, we first inspected the scans for artifacts induced by head movements. We found no corruption from the image using standard movement thresholds and hence kept all data for further processing. All diffusion-weighted images were processed based on the method used in our previous study (Lee Masson, et al., 2016b) so as to facilitate comparison across the two studies: in brief, the preprocessing procedure included eddy-current distortions correction using FSL from the FMRIB software library (Jenkinson, et al., 2012), gradient table rotation (Leemans and Jones, 2009), skull removal using FSL/BET, up-sampling the DWI data to 1mm-isotropic resolution, and standard denoising using Dipy ((Garyfallidis, 2014 \#136), http://nipy.org/dipy). After preprocessing, we fitted the diffusion tensor using Dipy to calculate the FA and $\mathrm{AD}$ of the TOIs for the later analysis. For the whole brain tractogram, we estimated the fiber orientation distribution function (fODF) using a spherical deconvolution method (harmonic order 8, FA > 0.7), followed by streamline deterministic tractography on the fODF with multiple seeds (20 seeds/voxel).

\section{Selection of functional ROIs (fROIs) and functionally defined tracts of interest (fTOIs)}

To extract fTOIs, we used fROIs from independent fMRI runs. Both visual and haptic fROIs were extracted from the well-established standard functional localizer runs made to obtain shape-selective brain regions (Bodegard, et al., 2001; Kanwisher, et al., 1997). Both localizer runs are identical to that used in our previous study (Lee Masson, et al., 2016a): intact and scrambled images of objects (including every-day objects and unfamiliar shapes) were shown in a rapid presentation paradigm for the visual localizer run that consisted of 16 randomized blocks, with each block presenting 15 images for $1 \mathrm{~s}$. The haptic localizer run consisted of shape blocks (using hand-held objects, such as spoon, cup and 3D novel shapes) and texture blocks (using cardboard-mounted pieces of material, such as wood, paper, or velvet cloth). During eight randomized blocks (four shape and four texture), participants explored the stimuli handed to them by an experimenter with their dominant hand for $6 \mathrm{~s}$ each. In both localizers, one-back tasks were given to participants to ensure continued attention.

Functional imaging data were analyzed using the Statistical Parametric Mapping software package (SPM8, Wellcome Department of Cognitive Neurology, London, UK). Images were corrected for slice timing, realigned to the mean image for the purpose of head movement correction, normalized to the Montreal Neurological Institute (MNI) template (re-sampling size of $2 \times 2 \times 2 \mathrm{~mm}^{3}$ ), and spatially smoothed (full-width at half-maximum $6 \mathrm{~mm}$ Gaussian kernel). After the preprocessing steps, a 
standard general linear model (GLM) was fitted between different conditions after adjusting for the effects of six head motion parameters obtained from the realignment procedure. Lastly, to yield group results, a random-effect analysis was implemented on individual contrast images (objects vs. scrambled for the visual contrast and objects vs. texture for the haptic contrast) with a one-sample t-test for statistical inference. Here, less stringent thresholds $(\mathrm{p}<0.001$, uncorrected) were applied to both functional localizer runs. To extract the sufficient amount of white matter streamlines using ROIs located in the grey matter area, the sizes of ROIs had to be large enough to include superficial white matter areas. This method is in line with several studies using uncorrected values from functional ROIs that extract fILF, for example (Gschwind et al, 2012; Gomez et al, 2015). With this threshold, group shape-selective regions were delineated as clusters for both sensory modalities in the following analyses. Since haptic exploration was done using the right (dominant) hand and since previous findings have also implicated only contra-lateral pathways (that is, left ILF, left SLF ft for visuo-haptic shape processing, see Lee Masson, 2016b), the ipsilateral (right) hemisphere was masked out for the selection of fROIs and we only report relevant activation in the left hemisphere.

Visual selective areas included inferior occipital gyrus (IOG), inferior temporal gyrus (ITG), fusiform gyrus (FG), middle temporal gyrus (MTG), angular gyrus (AG) and precuneus ( $\mathrm{PrCu})$. The postcentral gyrus (PostCG), precentral gyrus (PreCG), superior parietal lobule (SPL), and IOG were included as haptic object selective areas based on this analysis.

For the extraction of fTOIs, we selected two main regions based on the activation level of each cluster for each modality as fROIs. The idea behind this is to use these two regions as a pair of fROIs to delineate the white matter tracks (Gomez, et al., 2015; Greenberg, et al., 2012). These two main fROIs were used as pass-through-points rather than as end-points. Since the fROIs were adjacent to each other, the seed-target method was not suitable in order to extract the long streamlines that may play an important role in haptic processing (Figure 1). Importantly, we know that object shape processing is not independent from the processing of other low level physical properties, such as orientation, curvature and size that will require the involvement of other lower-level cortical areas. For instance, although it is known that LOC is involved in visual shape processing, it is neither start nor end point of that process (Lee Masson, et al., 2016a). Similarly, functional connectivity studies (e.g., Deshpande et al., 2008) claimed that haptic shape processing seemed to require a complex network with motor, 
somatosensory, frontal, and even higher-level visual areas, hence necessitating a broader approach to our white-matter analysis.

The selected visual functional ROIs were located in the posterior visual area (peak coordinate $\mathrm{x}$ y $\mathrm{z}=$ $45,-73,-2$; number of selected voxels $=1086$ ) and the anterior ventral part of visual area (peak coordinate $\mathrm{x} \mathrm{y} \mathrm{z}=-41,-43,-21$; number of selected voxels $=754)$ within the occipital lobe and temporal lobe respectively (Figure 1). Haptic functional ROIs were located in the PreCG (peak coordinate $\mathrm{x} \mathrm{y} \mathrm{z}=-32,-14,+57$; number of selected voxels $=1097$ ) and in the PostCG (peak coordinate $\mathrm{x} \mathrm{y} \mathrm{z}=-40,-34,+53$; number of selected voxels $=1474)$ in the motor and somatosensory areas respectively (Figure 1).

Using FSL, the selected fROIs were dilated twice so as to encompass the white matter areas, followed by its deformation from the MNI space to individual native diffusion spaces with the previously obtained FLIRT and ANTS transformations.

Each fTOI was therefore extracted based on the streamlines belonging to the proper anatomicallydefined TOI and requiring passage through its two fROIs. This method ensures that streamlines of the functionally-defined tracks are a subset of their anatomically-defined counterparts: all tracks of fILF, for example, pass through the two visual fROIs, but still belong to anatomically-defined ILF (aILF). Similarly, the streamlines of fSLF $f t$ and fSLF $f$ th that pass through the two haptic ROIs are parts of their respective anatomically-defined SLFs (aSLF_ft and $\left.\mathrm{aSLF}_{-} \mathrm{fp}\right)$. Based on the ROI-definition of Zhang et al., (2010), we built functionally-defined tracks situated within the anatomically relevant WM tracks (see details on how we respected anatomical restrains of the fTOIs in the supplementary materials (SM)). The extracted streamlines as fTOIs were used as a mask to compute the microstructural matrices (FA and AD). Visualization was made using TrackVis (Wang, et al., 2007).

\section{Analysis of sub-tracks of fTOIs}

After extracting fTOIs, we also analyzed the location of the ending points of each track and counted the streamlines between the subparts of various cortical regions. For example, streamlines connecting one ending point in IOG and another ending point in ITG were considered as constituting a sub-track of fILF. We evaluated how frequent each sub-track occurs across participants to examine the reliability of the sub-track within fTOIs. We also measured how much each sub-track was able to explain a particular fTOI by calculating the percentage of the total number of streamlines of each track. 
For the fILF, we extracted the streamlines between one ending point in the occipital lobe (among IOG, MOG, SOG, lingual gyrus (LG) and cuneus $(\mathrm{Cu})$ ) and another ending point in the temporal lobe (among ITG, MTG, STG, FG, temporal pole (TP)). For the $\mathrm{fSLF}_{-} \mathrm{ft}$, the streamlines included one ending point in frontal lobe (among IFG, MFG, SFG, lateral frontal orbital gyrus (LFOG), the medial frontal orbital gyrus (MFOG), gyrus rectus (GR), PreCG) and another ending point in the temporal lobe (among ITG, MTG, STG, FG, TP). Lastly, in case of $\mathrm{fSLF}_{-}$, the streamlines connecting one ending point in the frontal lobe (among IFG, MFG, SFG, LFOG, MFOG, GR, PreCG) with another ending point in either $\mathrm{AG}$ or supramarginal gyrus $(\mathrm{SMG})$ were extracted.

\section{Statistical Analysis}

First, we measured the number of required training blocks for each participant, followed by a ShapiroWilk test to verify distribution of normality. The average accuracy of the last two blocks was compared with that of the first two blocks using paired t-tests to check whether haptic performance improved during the training session for the participants. Subsequently, multiple linear regression analysis was performed with one of the microstructure scalar values (FA or $\mathrm{AD}$ ) of each fTOI as dependent variable based on haptic shape categorization performance, age, total intracranial volume (TIV), and head motion as regressors. The latter three variables were introduced as nuisance covariates. The SPM voxel-based morphometry (VBM8) toolbox was used for the calculation of TIV, with head motion measured by calculating the volume-by-volume translations and rotations from the affine preprocessing step (Yendiki, et al., 2014). Our particular choice of nuisance covariates was based on the information by previous studies that showed direct associations with microstructural WM characteristics, including FA (e.g., age with axonal and myelin degeneration (Abe et al., 2008; Zhang et al., 2010) and greater head movements with lower FA/AD (Yendiki et al., 2014)). All raw variables were converted to z-scores. In case of task performance, the z-scores were multiplied by $(-1)$ such that a high number of training blocks was labeled as "poor performance". Residuals were calculated by subtracting the predicted value from the observed value divided by the estimated standard deviation. Standardized residuals higher than 2 or lower than -2 were considered as outliers and excluded from the regression model. Lastly, Bonferroni correction for multiple comparisons was implemented with respect to the number of concerned fTOIs for the regression analyses. Statistical analyses were performed using MATLAB (R2015a, The Mathworks, Natick, MA, USA) built-in functions and JMP software (SAS, Cary, USA, version 9.0). 


\section{Results}

\section{Haptic categorization training}

Participants on average needed $13.6(\mathrm{SD}=6.4)$ blocks to finish the haptic categorization task. Importantly, the results show that large individual variability exists in the number of training blocks required to reach the criterion (range: 2 blocks indicating fast learners -20 blocks for slow learners). It is noteworthy that 13 out of 39 participants were not able to finish within the allotted 20 blocks. During the final two blocks (a total of 16 presented objects), however, accuracies for these participants were on average around $80 \%$, indicating performance much higher than chance level. Indeed, even for these 13 participants, the mean performance of the last two training blocks (77.9\%) was significantly higher than that of the first two blocks $(57.7 \%, \mathrm{t}(12)=3.38, \mathrm{p}=0.005)$, indicating considerable progress in category learning. Therefore, all 39 participants were included in the further analysis. The performance distribution overall showed a marked asymmetry, hence rejecting the null-hypothesis for a normal distribution $(\mathrm{p}<0.001)$. Due to this reason, we used $z$-standardized performance scores for the further analysis.

Task performance and microstructural metrics of the fTOIs and their sub-tracks

Distribution of fTOIs and their sub-tracks 
Figure 2 shows three fTOIs with respect to the location of each anatomically-defined TOI. Due to the additional constraints imposed by the visual functional ROIs in the ventrolateral part of the visual pathway, fILF is located in the ventrolateral part of aILF (See Figure 2-a). In the case of SLFs, both $\mathrm{fSLF}_{-\mathrm{ft}}$ and $\mathrm{fSLF}_{-}$are located in the dorsal part of $\mathrm{aSLF}_{-} \mathrm{ft}$ and $\mathrm{aSLF}_{-}$fp (See Figure 2-b and 2-c).

We also assessed the frequency of occurrence and the number of streamlines connecting a pair of regions to describe each sub-track. Here, we only report the sub-tracks contributing for more than 10 percent of a functionally-defined track. Table 1 describes the detailed information of anatomical, functional, and sub-tracks of each white matter pathway (ILF, SLF_ft and SLF_fp), including frequency of occurrence, group mean of the number of counted streamlines, the percentage of total streamlines of a certain track, and the scalar values (FA and $\mathrm{AD})$.

As shown in Table 1, 75\% of fILF was composed of four different sub-tracks, including the sub-track between FG and MOG, the sub-track between ITG and MOG, the sub-track between FG and IOG, and the sub-track between ITG and IOG (See Figure 3-a). In the case of fSLF $f$ t, the sub-tracks between MTG and IFG, MTG and MFG, and MTG and PreCG compose around 84\% (See Figure 3-b). Approximately $70 \%$ of $\mathrm{fSLF}_{-}$p is made up of three sub-tracks, including the sub-track between AG and MFG, the sub-track between AG and PreCG, and the sub-track between SMG and PreCG (See Figure 3-c).

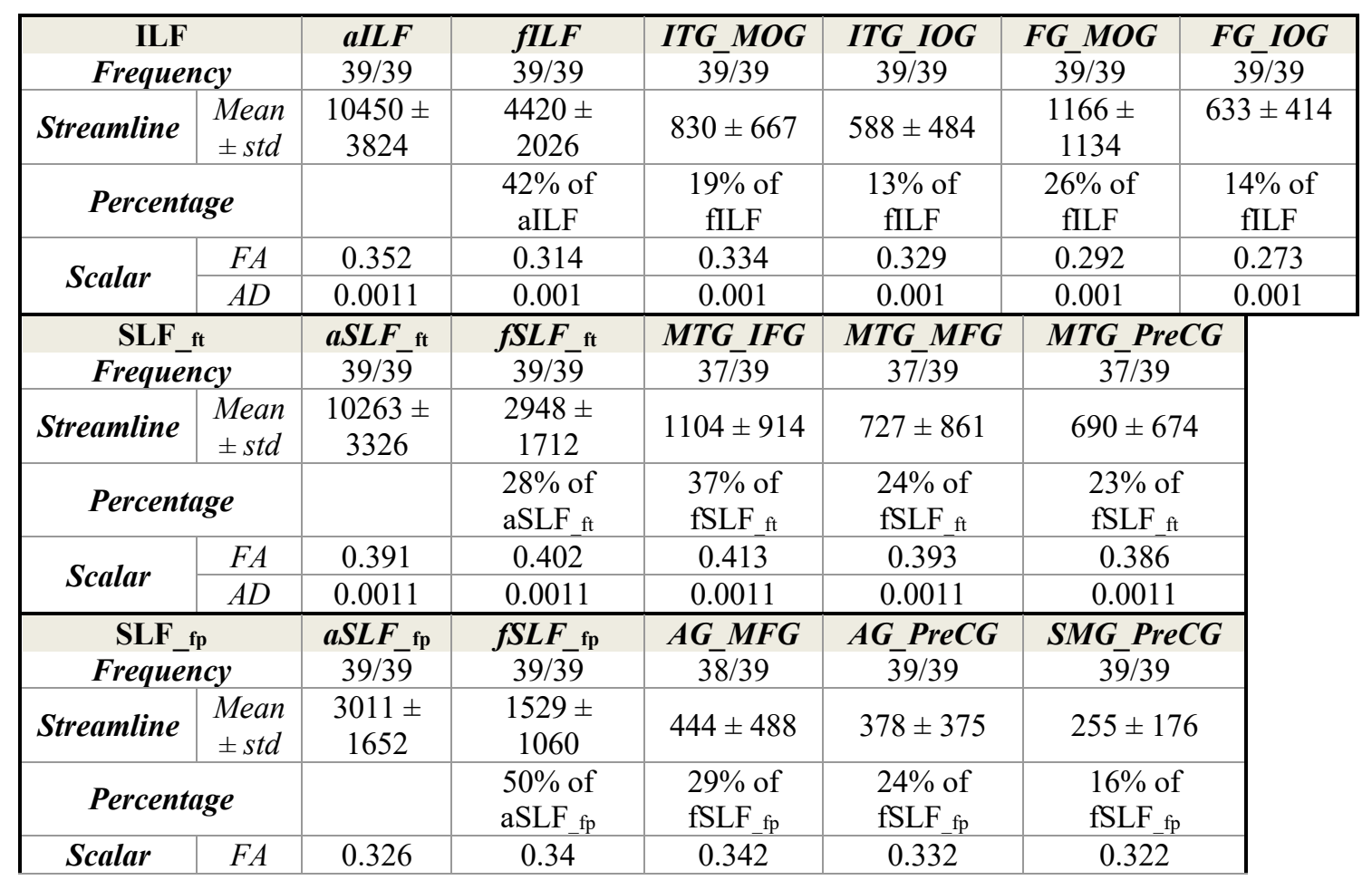




\begin{tabular}{|l|l|l|l|l|l|l|}
\hline & $A D$ & 0.001 & 0.001 & 0.001 & 0.001 & 0.0011 \\
\hline
\end{tabular}

Table 1 Each first row contains the frequency of occurrence for all tracks across participants. The number of streamlines is reported as group mean with standard deviation. The percentage value indicates the percentage of total streamlines of a certain track that make up each sub-track. In addition, both FA and AD are shown for each track.

\section{Task performance and microstructural metrics of the fTOIs and their sub-tracks}

We used the multiple regression analysis for each fTOI to examine correlations with individual variance in haptic training performance. The results indicate that individuals with high performance (fast learners) in haptic shape categorization had a larger mean AD of fSLF fp (estimated coefficients $=$ $0.4, \mathrm{p}=0.01,1$ outlier) in the left hemisphere after adjusting for effects of age, TIV and head motion (see also the results for purely anatomically-defined tracks as complementary findings in the SM).

Table 2 shows the overall results of each predictor's estimated coefficient, the coefficient of multiple determination for multiple regression $\left(\mathrm{R}^{2}\right)$, and its $\mathrm{p}$-value after removing outliers from the regression model based on calculation of the standardized residual. The number of outliers in each model is indicated in the last column of Table 2. Note that, a low coefficient of multiple determination $\left(\mathrm{R}^{2}\right)$ in this context should not be interpreted as low predictability caused by a weak association between the task performance and the scalar value of fTOIs. Including TIV, head motion and age in the regression model may easily degrade $\mathrm{R}^{2}$ when those nuisance covariates do not contribute to the model. Yet, even with this restriction, $34 \%$ of the response variable variations (mean AD of $\mathrm{SLF}_{-} \mathrm{p}$ ) could be explained by a linear model composed task performance and other covariates.

\begin{tabular}{|c|c|c|c|c|c|c|c|c|}
\hline $\begin{array}{c}\text { Track of } \\
\text { Interest }\end{array}$ & $\begin{array}{c}\text { Scalar } \\
\text { value }\end{array}$ & & & Performance & $T I V$ & $\begin{array}{c}\text { Head } \\
\text { Motion }\end{array}$ & Age & Outliers \\
\hline \multirow{3}{*}{$f I L F$} & & $R^{2}$ & $p$ & $\beta$ & $\beta$ & $\beta$ & $\beta$ & $\mathrm{N}$ \\
\hline & FA & 0.20 & 0.11 & 0.20 & 0.16 & -0.29 & -0.06 & 2 \\
\hline & $\mathrm{AD}$ & 0.20 & 0.12 & 0.22 & 0.30 & -0.12 & -0.18 & 2 \\
\hline \multirow[t]{2}{*}{$f S L F_{f t}$} & FA & 0.12 & 0.40 & 0.12 & 0.04 & -0.09 & 0.19 & 2 \\
\hline & $\mathrm{AD}$ & 0.04 & 0.83 & 0.18 & -0.01 & 0.04 & -0.10 & 1 \\
\hline \multirow{2}{*}{$f S L F_{f} p$} & FA & 0.18 & 0.16 & -0.06 & 0.43 & -0.17 & 0.10 & 1 \\
\hline & $\mathrm{AD}$ & 0.34 & $<0.01 *$ & $0.40^{*}$ & 0.00 & 0.14 & $-0.48 *$ & 1 \\
\hline
\end{tabular}

Table 2 Each row illustrates separate regression model based on different fTOI and their scalar values after removing potential outliers (based on the standardized residual exceeding an absolute value more than 2 ). $\mathrm{R}^{2}$ represents the coefficient of multiple determination and $p$ shows its significance level based on an $F$ test. The estimated coefficient $(\beta)$ indicates the slope of the regressor. The asterisk indicates that the results are significant following Bonferroni correction.

Lastly, we further refined this analysis to include the different sub-tracks of fSLFs. Among sub-tracks from $\mathrm{PSLF}_{\mathrm{ft}}$, we found significant regression weights for the mean FA (estimated coefficients $=0.45$, $\mathrm{p}=0.004,2$ outliers) and $\mathrm{AD}$ (estimated coefficients $=0.47, \mathrm{p}=0.005,1$ outlier) of tracks between 
MTG and IFG, the mean FA (estimated coefficients $=0.42, \mathrm{p}=0.004,1$ outlier) and AD (estimated coefficients $=0.49, \mathrm{p}=0.002$ ) of tracks between MTG and PreCG. We also found significant regression weights for the mean $\mathrm{AD}$ of tracks between $\mathrm{AG}$ and PreCG (estimated coefficients $=0.44, \mathrm{p}$ $=0.006,1$ outlier) as a part of local sub-tracks of PSLF_fp, again after controlling for nuisance covariates and outliers. Overall, higher haptic performance goes along with a higher mean $\mathrm{AD} / \mathrm{FA}$ of functionally-defined white matter tracks, including $\mathrm{SLF}_{-\mathrm{fp}}$, and local sub-tracks between MTG and IFG, between MTG and PreCG, and between AG and PreCG. Figure 4 demonstrates the slopes of each linear regression model between adjusted microstructural matrices and haptic performance.

Importantly, the results of the present study testing haptic performance without prior visual knowledge (training) does not show any association between task performance and microstructural characteristics of fILF or any of its sub-tracks.

Visuo-haptic cross-modal performance and microstructural matrices of fILF, fSLFs and their sub-tracks

The purpose of re-visiting our previous study is to compare haptic shape categorization performance without visual knowledge (current study) and with visual knowledge (previous study). In that study, we showed that microstructural characteristics of $\mathrm{aSLF}_{-} \mathrm{ft}$ and aILF reflect the cross-modal haptic shape categorization performance after visual training, emphasizing the role of left ILF and its potential connection to visual imagery during haptic processing (Lee Masson, et al., 2016b).

To compare these results with the ones of the present study, we extracted scalar values of functionallydefined tracks and their sub-tracks from the previous DWI data. Then, we calculated beta estimates of all extracted tracks using multiple regression analysis to identify their engagement in visuo-haptic cross-modal learning. As shown in Table 3 and Figure 5, the results illustrated that haptic performance after visual training shows significantly negative regression weights for the mean FA (estimated coefficients $=-0.4, \mathrm{p}=0.01,1$ outlier) and $\mathrm{AD}$ (estimated coefficients $=-0.45, \mathrm{p}=0.006)$ of left fILF after controlling for nuisance covariates.

Lastly, we also evaluated estimated coefficients of performance on local sub-tracks of fILF and ASLFs to examine which sub-tracks can be predicted by visuo-haptic performance. The results do show tendencies for the mean FA (estimated coefficients $=0.36, \mathrm{p}=0.02,1$ outlier) and $\mathrm{AD}$ (estimated 
coefficients $=0.35, \mathrm{p}=0.04,1$ outlier) of AG_PreCG pathway, AD of SMG_PreCG pathway (estimated coefficients $=0.37, \mathrm{p}=0.02,1$ outlier), and FA of the ITG_IOG pathway (estimated coefficients $=-0.36, \mathrm{p}=0.04)$ to be associated with cross-modal categorization performance.

\begin{tabular}{l|cccccccc}
\multicolumn{1}{c}{$\begin{array}{c}\text { Track of } \\
\text { Interest }\end{array}$} & $\begin{array}{c}\text { Scalar } \\
\text { value }\end{array}$ & & & Performance & TV & $\begin{array}{c}\text { Head } \\
\text { Motion }\end{array}$ & Age & Outliers \\
\hline \multirow{3}{*}{$f \boldsymbol{f L F}$} & & $R^{2}$ & $p$ & $\beta$ & $\beta$ & $\beta$ & $\beta$ & $\mathrm{N}$ \\
& FA & 0.28 & 0.03 & $-0.40^{*}$ & -0.18 & 0.18 & -0.15 & 1 \\
$f S L F_{f t}$ & AD & 0.36 & $<0,01^{*}$ & $-0.45^{*}$ & -0.26 & 0.17 & -0.31 & 0 \\
& FA & 0.17 & 0.21 & -0.26 & 0.22 & -0.03 & 0.12 & 1 \\
$f S L F_{f p}$ & AD & 0.02 & 0.98 & -0.05 & -0.01 & -0.00 & -0.1 & 2 \\
& FA & 0.07 & 0.66 & 0.20 & 0.14 & 0.07 & 0.02 & 0 \\
& AD & 0.28 & 0.03 & 0.38 & -0.15 & -0.16 & -0.22 & 0
\end{tabular}

Table 3 Each row illustrates a separate regression model based on a different TOI and their scalar values after removing potential outliers (based on the standardized residual exceeding an absolute value more than 2 ). $\mathrm{R}^{2}$ represents the coefficient of multiple determination and $\mathrm{p}$ its significance level based on an F-test. The estimated coefficient $(\beta)$ indicates the slope of the regressor. The asterisk indicates that the results are significant following Bonferroni correction.

\section{Visual performance and microstructural matrices of fILF, fSLFs and their sub-tracks}

Our previous study also showed that a higher mean FA of both $\mathrm{aSLF}_{-} \mathrm{ft}$ and ventral-occipital fasciculus (VOF) was correlated with fast visual learning speed (Lee Masson, et al., 2016b). Here, we calculated beta estimates for all functionally-defined tracts in relation to visual learning speed ${ }^{1}$. The results confirm a correlation with the mean FA in left $\mathrm{fSLF}_{-} \mathrm{ft}$ (Table 4).

\begin{tabular}{|c|c|c|c|c|c|c|c|c|}
\hline $\begin{array}{l}\text { Track of } \\
\text { Interest }\end{array}$ & $\begin{array}{c}\text { Scalar } \\
\text { value }\end{array}$ & & & Performance & $T V$ & $\begin{array}{c}\text { Head } \\
\text { Motion }\end{array}$ & Age & Outlier \\
\hline \multirow{3}{*}{ fILF } & & $R^{2}$ & $p$ & $\beta$ & $\beta$ & $\beta$ & $\beta$ & $\mathrm{N}$ \\
\hline & FA & 0.13 & 0.38 & -0.01 & -0.21 & 0.17 & -0.05 & 2 \\
\hline & $\mathrm{AD}$ & 0.25 & 0.06 & -0.07 & -0.31 & 0.23 & -0.20 & 1 \\
\hline \multirow[t]{2}{*}{$f S L F_{f} f t$} & FA & 0.29 & 0.03 & $0.39 *$ & 0.15 & 0.04 & 0.21 & 1 \\
\hline & $\mathrm{AD}$ & 0.17 & 0.25 & 0.32 & -0.05 & 0.04 & -0.08 & 2 \\
\hline \multirow[t]{2}{*}{$f S L F_{f} p$} & FA & 0.16 & 0.25 & 0.35 & 0.09 & 0.03 & -0.06 & 1 \\
\hline & $\mathrm{AD}$ & 0.16 & 0.23 & 0.07 & -0.09 & -0.11 & -0.34 & 0 \\
\hline
\end{tabular}

Table 4 Each row illustrates a separate regression model based on a different TOI and their scalar values after removing potential outliers (based on the standardized residual exceeding an absolute value more than 2 ). $\mathrm{R}^{2}$ represents the coefficient of multiple determination and $\mathrm{p}$ its significance level based on an F-test. The estimated coefficient $(\beta)$ indicates the slope of the regressor. The asterisk indicates that the results are significant following Bonferroni correction.

Among the various sub-tracks, we found no significant results after correcting for multiple comparisons. Nonetheless, visual performance shows a tendency for positive regression weights for the

\footnotetext{
1 We leave out the VOF in the present paper since our previous study did not show any haptic or visuo-haptic shape processing involvement (Lee Masson et al., 2016b).
} 
mean FA of the sub-tracks between MTG and IFG (estimated coefficients $=0.38, p=0,04$ ) and for the mean FA of the sub-tracks between SMG and PreCG (estimated coefficients $=0.35, \mathrm{p}=0,02,1$ outlier).

\section{Discussion}

The aim of the current study was to define the WM pathways involved in higher-level haptic processing. To address this question, we measured learning speed in a difficult shape categorization task and utilized this behavioral data to identify performance correlates in several major WM bundles. Importantly, pathways were extracted functionally with the help of functional imaging data identifying modality-specific, shape selective areas. Additionally, sub-tracks connecting different cortical areas were also extracted from the functionally-defined tracks. For each track, its correlation with the haptic performance was then determined. Finally, we re-analyzed previous data in order to compare and contrast performance correlates in different modality-specific conditions.

The present study is the first to identify clear WM involvements for object processing in touch: by combining in vivo DWI and fMRI data, our findings revealed that individual performance differences in categorization performance were significantly correlated with microstructural characteristics of several WM tracks passing through haptic shape-selective regions. The current findings on the broad involvements of multiple WM pathways on haptic shape perception appears in line with the earlier functional connectivity studies that demonstrated the high complexity of the neural networks that were involved in processing haptically explored shape features, suggesting multi-directional (even lateral) cross-talks among the cortical regions (Deshpande et al., 2008; Peltier et al., 2007).

Extraction of shape features by touch requires both cutaneous and kinaesthetic inputs obtained from exploratory procedures, including hand enclosure and contour following for global and precise shape processing respectively (Lederman and Klatzky 2009). As proprioceptive inputs alone would not be able to convey the perception of the touched object, cutaneous inputs without proprioceptive information would present solely the surface information to the skin without delivering the shape information. Thus, haptic shape representation can be only formed by (serial) movement of the finger tips sweeping along the contour or surface of the objects in different directions while extracting touch information. Such a tight coupling between the action (motor planning and execution) from the kinaesthetic inputs and tactile perception from cutaneous inputs on haptic shape recognition task is indeed reflected in the current findings: specifically, the involvements of fSLF $f_{\mathrm{p}}$ and its sub-track (AG- 
PreCG), which connects the frontal area with the posterior parietal cortex while traversing haptic selective regions, namely PreCG and PostCG, highlight that high-level somatosensory areas in the posterior parietal lobe work in concert with motor/premotor areas in the frontal lobe for the purpose of planning and executing fine hand (finger) movements to optimally extract the relevant macrogeometric features during haptic shape categorization. Previous functional neuroimaging evidence demonstrating increased neural activation (Bodegard, et al., 2001; Reed, et al., 2004) and detailed neural shape representations (Lee Masson, et al., 2016a) in the premotor and the parietal lobe areas during the haptic shape recognition task is also in line with our current findings. Moreover, the bidirectional connection between (pre)motor and posterior part of somatosensory areas has already been observed by other DWI studies, claiming SLF $_{-}$(or SLFII) to subserve reaching and grasping processes (Vry, et al., 2015).

In spite of the contribution of fine motor coordination, haptic shape processing also seems to involve the visual modality. Indeed, for more than a decade, neuroimaging studies have suggested that haptic shape representations themselves can be multisensory in nature. For example, demonstrating haptic shape selectivity in the LOC that previously was only seen as part of the visual processing pipeline (Amedi et al. 2001; Amedi et al. 2002; James et al. 2002; Kassuba et al. 2013) spurred debates about the role of visual imagery in haptic shape perception. A few studies investigated whether visual imagery played a role in haptic shape processing, revealing that familiar shape processing via haptic modality shares similar neural network with visual imagery task (Zhang et al. 2004; Deshpande et al. 2010). In contrast, evidence on LOC activation found in blind patients who could not exhibit visual imagery during haptic shape processing challenged the role of that particular area related to visual imagery (Amedi et al. 2010).

In the present study, however, we did not observe fILF correlates - the track that contains early- to high-level visual areas as well as further visually-selective regions (IOG/LOC and ITG). This is in contrast to our previous study (Lee Masson, 2016b) and the data re-analyzed here in which visuohaptic transfer performance showed clear correlations in both anatomically- and functionally-defined ILF. Taken together, these results indicate that ILF may relay visual information (if available from prior visual learning) during haptic shape processing for the purpose of facilitating haptic performance. Interestingly, this interpretation is also consistent with the data from our previous functional neuroimaging study which showed extended neural correlates of shape representations in the ventral 
stream, but only if the objects had been previously experienced in the visual modality (Lee Masson, et al., 2016a). Additional neuropsychological evidence for this view comes from a recent study (Snow et al. 2015) that demonstrated that lesions in LOC did not cause tactile agnosia, whereas visual agnosia was severe, suggesting that LOC is not necessary for haptic shape processing per se. Instead, LOC could be activated by visual imagery or by feedback from other brain regions to achieve more efficient or accurate performance during the haptic shape recognition task.

Lastly, multiple sub-tracks (MTG-IFG and MTG-PreCG) belonging to the $\mathrm{ASLF}_{-} \mathrm{ft}$ were identified as related to haptic performance. Previous functional neuroimaging studies that implicated PreCG, AG, MTG and IFG in haptic shape processing (Binkofski, et al., 1999; Reed, et al., 2004; Stilla and Sathian, 2008) provide complementary evidence for our WM-related findings.

Concerning $\mathrm{SLF}_{-} \mathrm{ft}$, we conjecture that it may serve as supramodal pathway as evidenced by significant performance correlations in the visual, haptic, and visuo-haptic conditions based on the results of $\mathrm{aSLF}_{-} \mathrm{ft}$ (for the detailed results of $\mathrm{aSLF}_{-} \mathrm{ft}$ see $\mathrm{SM}$ ). The $\mathrm{SLF}_{-} \mathrm{ft}$ is a long, associative WM pathway connecting frontal, parietal and temporal lobes. Previous DWI studies have shown that the microstructure composition of this long track is associated with multiple perceptual functions such as spatial attention to features in object shape processing (Mayer and Vuong, 2014), visuo-spatial attention (Kantarci, et al., 2011), sensory-motor interaction (Vry, et al., 2015), visual shape learning, visuo-motor coordination, and visuo-haptic cross-modal transfer ability (Lee Masson, et al., 2016b). Our data indicates that $\mathrm{SLF}_{-}$may support supramodal shape information regardless of sensory modality.

In this context, one should note that a similar anatomical structure to the SLF_ft is referred to in other studies as the arcuate fasciculus (AF). When following the definition proposed by Zhang et al. (2010), the streamlines of SLF $\mathrm{ft}$ terminate in the frontal lobe and in the temporal lobe, bypassing a series of other ROIs (e.g., the occipital lobe). In contrast, AF is defined from the streamlines connecting Broca's area in the frontal lobe with Wernicke areas in the temporal lobe (Catani 2005). Although the exact definitions are different, their resulting streamlines have considerable overlap. Given its ROI-definition, the AF appears to be more often cited in studies related to language function (e.g. Catani et al, 2007), whereas the SLF(III) is mostly used in studies dealing with sensory-processing related functions, such as spatial attention (e.g. Thiebaut de Schotten et al., 2011). To be consistent with our previous study 
(Lee Masson et al, 2016b) and considering the task (sensory processing) used in this study, we selected the definition in Zhang et al. (2010) to determine our WM track.

In summary, as we hypothesized, fILF and fSLF $_{-}$were dissociated for shape processing based on the availability of prior visual knowledge. Moreover, $\mathrm{fSLF}_{-} \mathrm{ft}$ as a subpart of $\mathrm{SLF}_{-} \mathrm{ft}$ was found engaged in visual shape learning. The full data is summarized in figure 7 , reflecting both unisensory and multisensory processes in shape processing.

The novel findings on neuroanatomical correlates of haptic shape processing in the current study was realized by implementing a more advanced processing pipeline based on the fODF with a spherical deconvolution method (Farquharson, 2013) which enabled us to overcome methodological constrains when extracting rarely investigated WM tracks such as $\operatorname{SLF}_{-}$(pee the review paper of Wandell, 2016). Lastly, although the present study presents converging evidence for neuroanatomical correlates of visual and haptic task performance, further conditions, such as a haptic-to-vision transfer condition are yet to be investigated in more detail. Based on the data so far, we would conjecture $\operatorname{SLF}_{-}$ft to be involved as a supra-modal processing track and would also expect performance correlates with $\mathrm{SLF}_{-} \mathrm{fp}$ due to the possible usage of haptic (motor) imagery during the task given sufficient haptic training. It will be also interesting to complement the present line of research with studies on multisensory processing of familiar objects, as well as integration of other object properties, such as weight or texture to get a full understanding of how and where the brain represents and processes object information. 


\section{References}

Amedi, A., Jacobson, G., Hendler, T., Malach, R., Zohary, E. (2002) Convergence of visual and tactile shape processing in the human lateral occipital complex. Cereb Cortex, 12:1202-12.

Binkofski, F., Buccino, G., Posse, S., Seitz, R.J., Rizzolatti, G., Freund, H. (1999) A frontoparietal circuit for object manipulation in man: evidence from an fMRI-study. Eur J Neurosci, 11:3276-86.

Bodegard, A., Geyer, S., Grefkes, C., Zilles, K., Roland, P.E. (2001) Hierarchical processing of tactile shape in the human brain. Neuron, 31:317-28.

Coello, A.F., Duvaux, S., De Benedictis, A., Matsuda, R., Duffau, H. (2013) Involvement of the right inferior longitudinal fascicle in visual hemiagnosia: a brain stimulation mapping study: Case report. Journal of neurosurgery, 118:202-205.

Gaißert, N., Wallraven, C., Bülthoff, H.H. (2010) Visual and haptic perceptual spaces show high similarity in humans. Journal of Vision. $\mathrm{p} 2$.

Gomez, J., Pestilli, F., Witthoft, N., Golarai, G., Liberman, A., Poltoratski, S., Yoon, J., GrillSpector, K. (2015) Functionally defined white matter reveals segregated pathways in human ventral temporal cortex associated with category-specific processing. Neuron, 85:216-27.

Greenberg, A.S., Verstynen, T., Chiu, Y.-C., Yantis, S., Schneider, W., Behrmann, M. (2012) Visuotopic cortical connectivity underlying attention revealed with white-matter tractography. Journal of Neuroscience, 32:2773-2782.

Grossi, D., Soricelli, A., Ponari, M., Salvatore, E., Quarantelli, M., Prinster, A., Trojano, L. (2014) Structural connectivity in a single case of progressive prosopagnosia: the role of the right inferior longitudinal fasciculus. Cortex, 56:111-20.

Gschwind, M., Pourtois, G., Schwartz, S., Van De Ville, D., Vuilleumier, P. (2012) Whitematter connectivity between face-responsive regions in the human brain. Cerebral Cortex (New York, NY), 22:1564-1576.

James, T.W., Kim, S., Fisher, J.S. (2007) The neural basis of haptic object processing. Can J Exp Psychol, 61:219-29.

Jenkinson, M., Beckmann, C.F., Behrens, T.E.J., Woolrich, M.W., Smith, S.M. (2012) FSL. NeuroImage, 62:782-790.

Kantarci, K., Senjem, M., Avula, R., Zhang, B., Samikoglu, A., Weigand, S., Przybelski, S., Edmonson, H., Vemuri, P., Knopman, D. (2011) Diffusion tensor imaging and cognitive function in older adults with no dementia. Neurology, 77:26-34.

Kanwisher, N., Woods, R.P., Iacoboni, M., Mazziotta, J.C. (1997) A locus in human extrastriate cortex for visual shape analysis. J Cogn Neurosci, 9:133-42.

Klatzky, R.L., Lederman, S.J., Metzger, V.A. (1985) Identifying objects by touch: An "expert system". Perception \& Psychophysics, 37:299-302.

Lacey, S., Flueckiger, P., Stilla, R., Lava, M., Sathian, K. (2010) Object familiarity modulates the relationship between visual object imagery and haptic shape perception. Neuroimage, 49:1977-1990.

Lacey, S., Tal, N., Amedi, A., Sathian, K. (2009) A putative model of multisensory object representation. Brain Topogr, 21:269-74.

Lee Masson, H., Bulthé, J., De Beeck, H.P.O., Wallraven, C. (2016a) Visual and Haptic Shape Processing in the Human Brain: Unisensory Processing, Multisensory Convergence, and Top-Down Influences. Cerebral Cortex, 26:3402-3412.

Lee Masson, H., Wallraven, C., Petit, L. (2016b) "Can touch this": Cross-modal shape categorization performance is associated with microstructural characteristics of white matter association pathways. Human Brain Mapping.

Leemans, A., Jones, D.K. (2009) The B-matrix must be rotated when correcting for subject motion in DTI data. Magnetic Resonance in Medicine, 61:1336-1349. 
Liakakis, G., Nickel, J., Seitz, R.J. (2011) Diversity of the inferior frontal gyrus--a metaanalysis of neuroimaging studies. Behav Brain Res, 225:341-7.

Lombardo, M.V., Chakrabarti, B., Bullmore, E.T., Sadek, S.A., Pasco, G., Wheelwright, S.J., Suckling, J., Baron-Cohen, S., Consortium, M.A. (2010) Atypical neural selfrepresentation in autism. Brain, 133:611-624.

Mayer, K., Vuong, Q. (2014) TBSS and probabilistic tractography reveal white matter connections for attention to object features. Brain Struct Funct, 219:2159-2171.

Oishi, K., Faria, A., Jiang, H., Li, X., Akhter, K., Zhang, J., Hsu, J.T., Miller, M.I., van Zijl, P.C.M., Albert, M. (2009) Atlas-based whole brain white matter analysis using large deformation diffeomorphic metric mapping: application to normal elderly and Alzheimer's disease participants. Neuroimage, 46:486-499.

Oostra, K.M., Van Bladel, A., Vanhoonacker, A.C., Vingerhoets, G. (2016) Damage to Fronto-Parietal Networks Impairs Motor Imagery Ability after Stroke: A VoxelBased Lesion Symptom Mapping Study. Frontiers in behavioral neuroscience, 10.

Ortibus, E., Verhoeven, J., Sunaert, S., Casteels, I., de Cock, P., Lagae, L. (2012) Integrity of the inferior longitudinal fasciculus and impaired object recognition in children: a diffusion tensor imaging study. Dev Med Child Neurol, 54:38-43.

Peters, B.D., Ikuta, T., DeRosse, P., John, M., Burdick, K.E., Gruner, P., Prendergast, D.M., Szeszko, P.R., Malhotra, A.K. (2014) Age-related differences in white matter tract microstructure are associated with cognitive performance from childhood to adulthood. Biol Psychiatry, 75:248-56.

Reed, C.L., Caselli, R.J. (1994) The nature of tactile agnosia: a case study. Neuropsychologia, 32:527-539.

Reed, C.L., Shoham, S., Halgren, E. (2004) Neural substrates of tactile object recognition: an fMRI study. Hum Brain Mapp, 21:236-46.

Sarro, L., Agosta, F., Canu, E., Riva, N., Prelle, A., Copetti, M., Riccitelli, G., Comi, G., Filippi, M. (2011) Cognitive functions and white matter tract damage in amyotrophic lateral sclerosis: a diffusion tensor tractography study. American Journal of Neuroradiology, 32:1866-1872.

Sebanz, N., Knoblich, G., Stumpf, L., Prinz, W. (2005) Far from action-blind: Representation of others' actions in individuals with autism. Cognitive Neuropsychology, 22:433-454.

Shinoura, N., Suzuki, Y., Tsukada, M., Katsuki, S., Yamada, R., Tabei, Y., Saito, K., Yagi, K. (2007) Impairment of inferior longitudinal fasciculus plays a role in visual memory disturbance. Neurocase, 13:127-130.

Snow, J.C., Strother, L., Humphreys, G.W. (2014) Haptic shape processing in visual cortex. J Cogn Neurosci, 26:1154-67.

Stilla, R., Sathian, K. (2008) Selective visuo-haptic processing of shape and texture. Hum Brain Mapp, 29:1123-38.

Stoeckel, M.C., Weder, B., Binkofski, F., Buccino, G., Shah, N.J., Seitz, R.J. (2003) A frontoparietal circuit for tactile object discrimination: an event-related fMRI study. Neuroimage, 19:1103-14

Tavor, I., Yablonski, M., Mezer, A., Rom, S., Assaf, Y., Yovel, G. (2014) Separate parts of occipito-temporal white matter fibers are associated with recognition of faces and places. Neuroimage, 86:123-30.

Voineskos, A.N., Rajji, T.K., Lobaugh, N.J., Miranda, D., Shenton, M.E., Kennedy, J.L., Pollock, B.G., Mulsant, B.H. (2012) Age-related decline in white matter tract integrity and cognitive performance: A DTI tractography and structural equation modeling study. Neurobiology of Aging, 33:21-34.

Vry, M.S., Tritschler, L.C., Hamzei, F., Rijntjes, M., Kaller, C.P., Hoeren, M., Umarova, R., Glauche, V., Hermsdoerfer, J., Goldenberg, G., Hennig, J., Weiller, C. (2015) The ventral fiber pathway for pantomime of object use. Neuroimage, 106:252-63. 
Wallraven, C., Bülthoff, H.H., Waterkamp, S., van Dam, L., Gaißert, N. (2014) The eyes grasp, the hands see: Metric category knowledge transfers between vision and touch. Psychonomic bulletin \& review, 21:976-985.

Wang, R., Benner, T., Sorensen, A.G., Wedeen, V.J. (Diffusion toolkit: a software package for diffusion imaging data processing and tractography). In; 2007.

Yendiki, A., Koldewyn, K., Kakunoori, S., Kanwisher, N., Fischl, B. (2014) Spurious group differences due to head motion in a diffusion MRI study. Neuroimage, 88:79-90.

Zhang, Y., Zhang, J., Oishi, K., Faria, A.V., Jiang, H., Li, X., Akhter, K., Rosa-Neto, P., Pike, G.B., Evans, A. (2010) Atlas-guided tract reconstruction for automated and comprehensive examination of the white matter anatomy. Neuroimage, 52:1289-1301. 


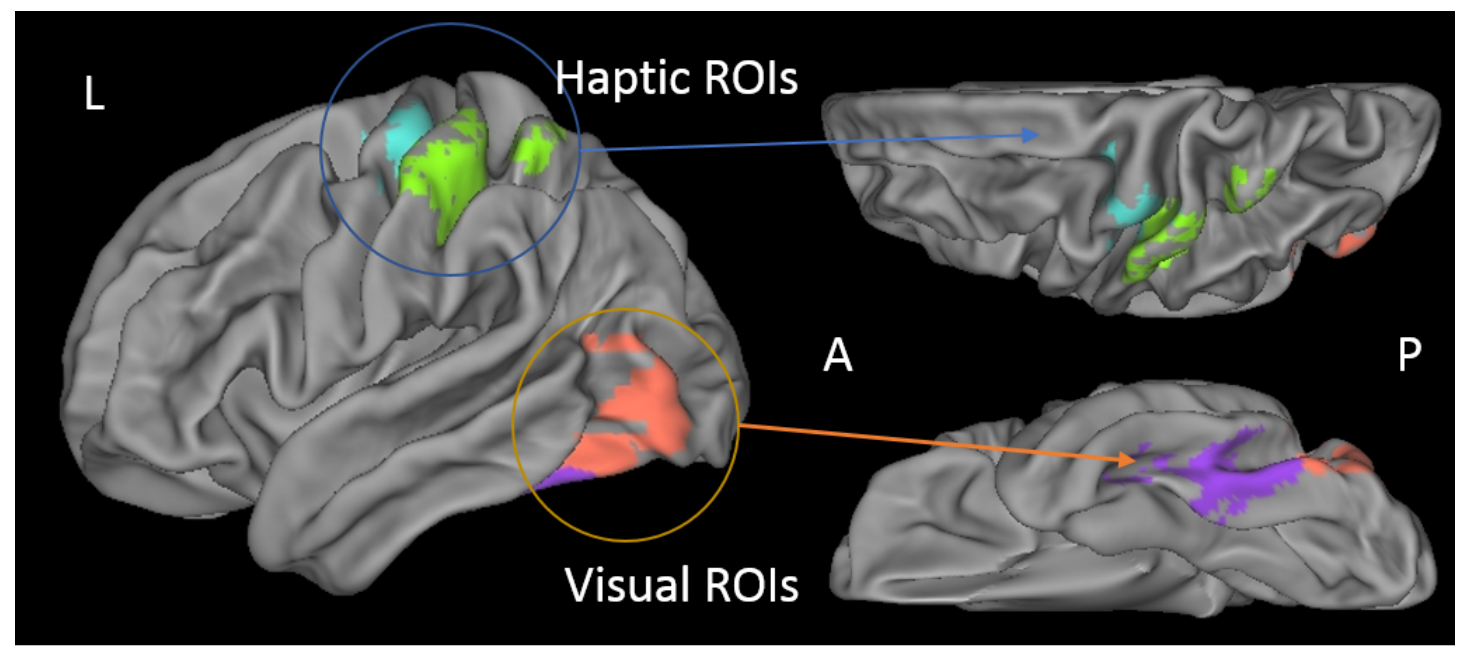

Fig. 1 Group results demonstrate object-selective activations from each sensory modality (as identified by the contrast [objects > scrambled (for vision), objects $>$ texture (for haptic)]), including IOG (orange) and ITG (purple) for vision and PreCG (cyan) and PostCG (light green) for haptic, in the left hemisphere (one sample t-test, P $>0.001$ uncorrected). L, A and P stand for left, anterior and posterior respectively. 


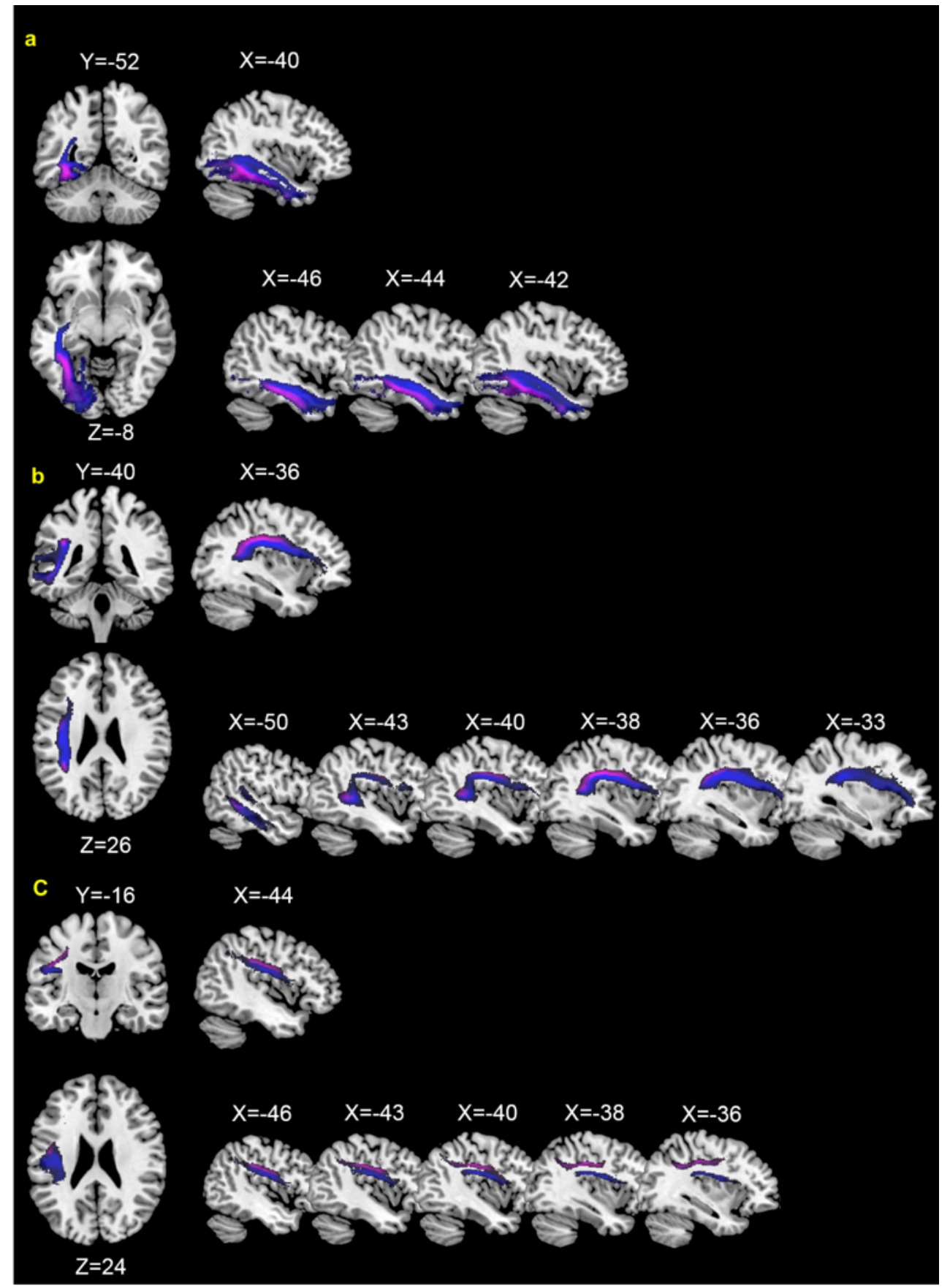

Fig. 2 Anatomically defined TOIs (blue) and functionally defined TOIs (pink) overlaid on the standard MNI template. ILF, $\mathrm{SLF}_{-} \mathrm{ft}$ and $\mathrm{SLF}_{-\mathrm{fp}}$ are shown in $\mathrm{a}, \mathrm{b}$, and $\mathrm{c}$ respectively. Note that functionally defined tracks are always located within the anatomically defined one. For visualization purposes, we transformed both tracks into MNI space so as to observe the relative location of each fTOI in the MNI space. All the sagittal slices were from the left hemisphere. MNI x, y, z coordinates were used to describe each slice of the brain. 


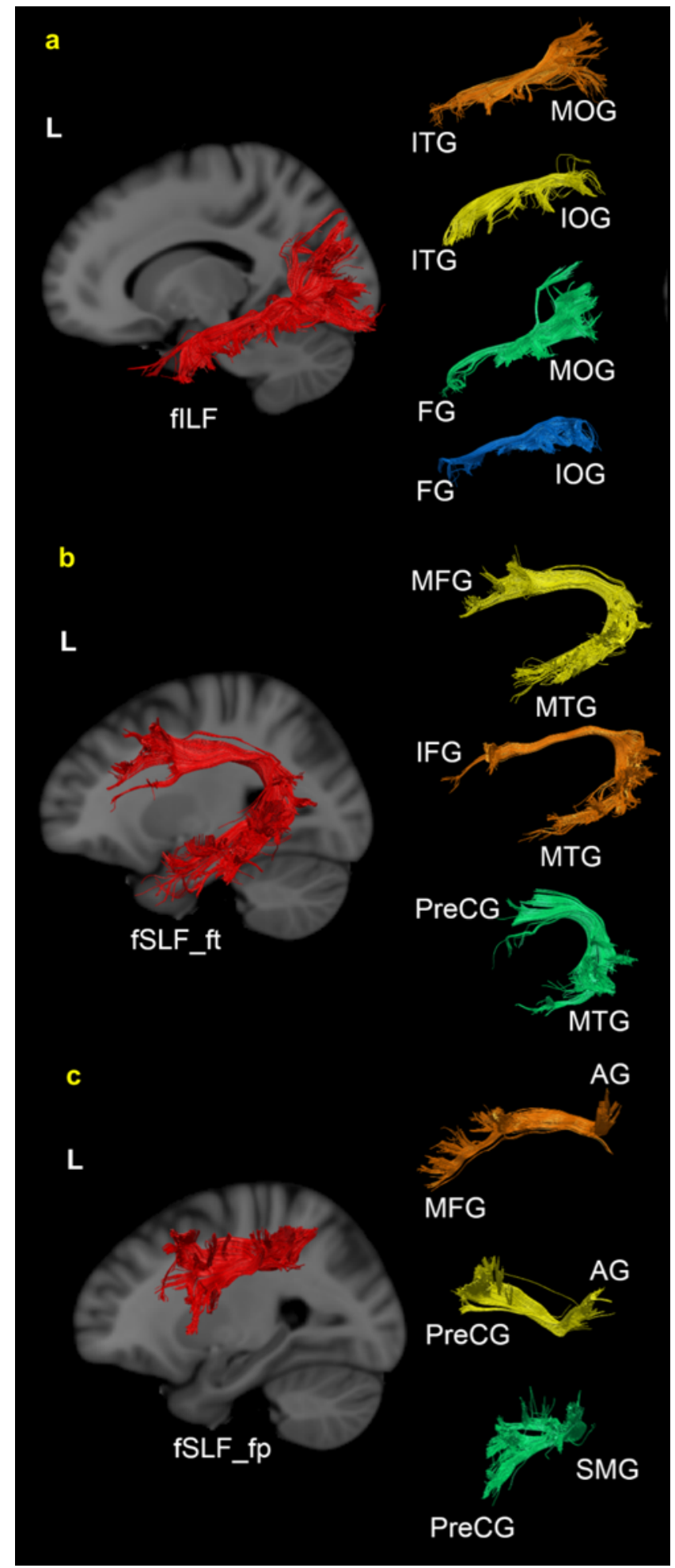

Fig. 3 Example of left fILF (a), fSLF_ft (b), fSLF_fp (c) and their sub-tracks extracted from the whole brain tractography of a representative participant. $\mathrm{L}=$ left hemisphere 

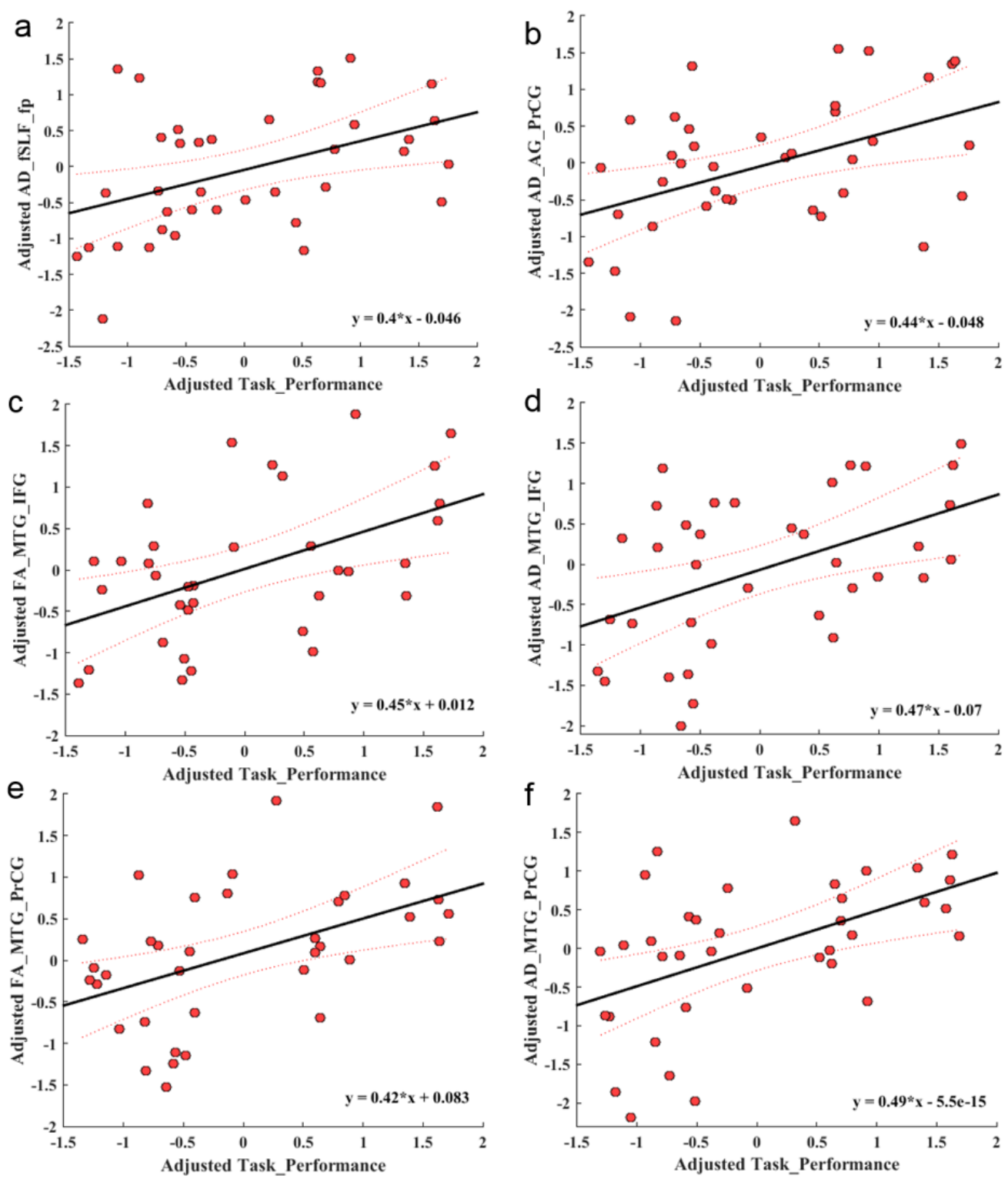

Fig. 4 Scatter plots show the fitted linear model (black line) and the equation for correlating haptic task performance with the scalar value of $f S L F_{f p}$ and sub-tracks of fSLFs (a. mean $\mathrm{AD}$ of $\mathrm{fSLF}_{\mathrm{fp}}$, b. mean $\mathrm{AD}$ of subtracks between AG and PreCG, c. mean FA of sub-tracks between MTG and IFG, d. mean AD of sub-tracks between MTG and IFG, e. mean FA of sub-tracks between MTG and PreCG, f. mean AD of sub-tracks between MTG and PreCG) after adjusting for all other variables and excluding outliers. Note that all variables are z- score standardized on both $\mathrm{x}$ - and $\mathrm{y}$-axis. Only significant results are shown in this figure. 

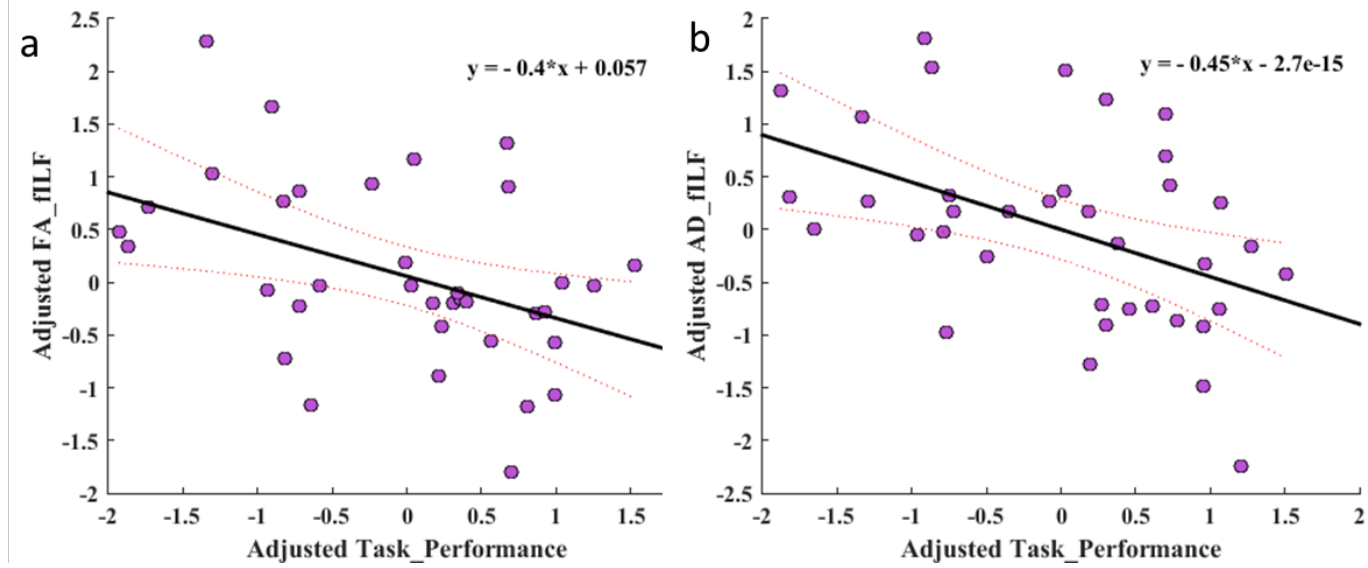

Fig. 5 Each scatter plot shows the fitted linear model (black line) and the equation for correlating visuo-haptic transfer performance with the mean FA of fILF(a) and the mean AD of fILF (b) after adjusting for nuisance covariates and outliers.

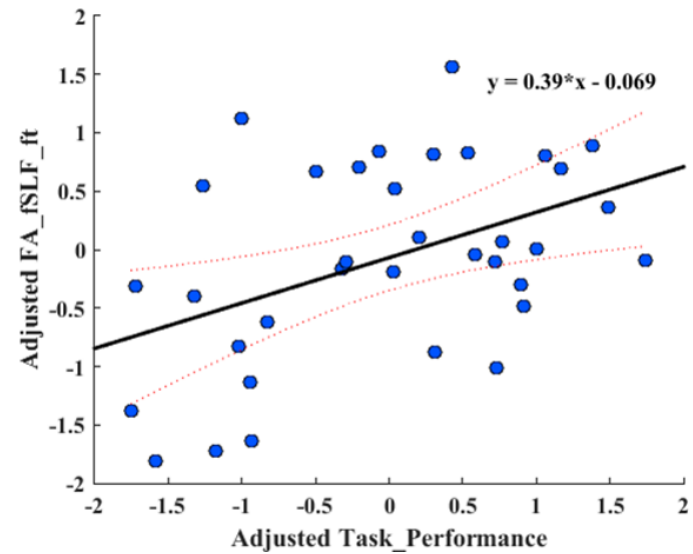

Fig. 6 Fitted linear model (black line) and the equation for correlating visual task performance with the scalar value of fSLF_ft after adjusting for nuisance covariates and outliers 


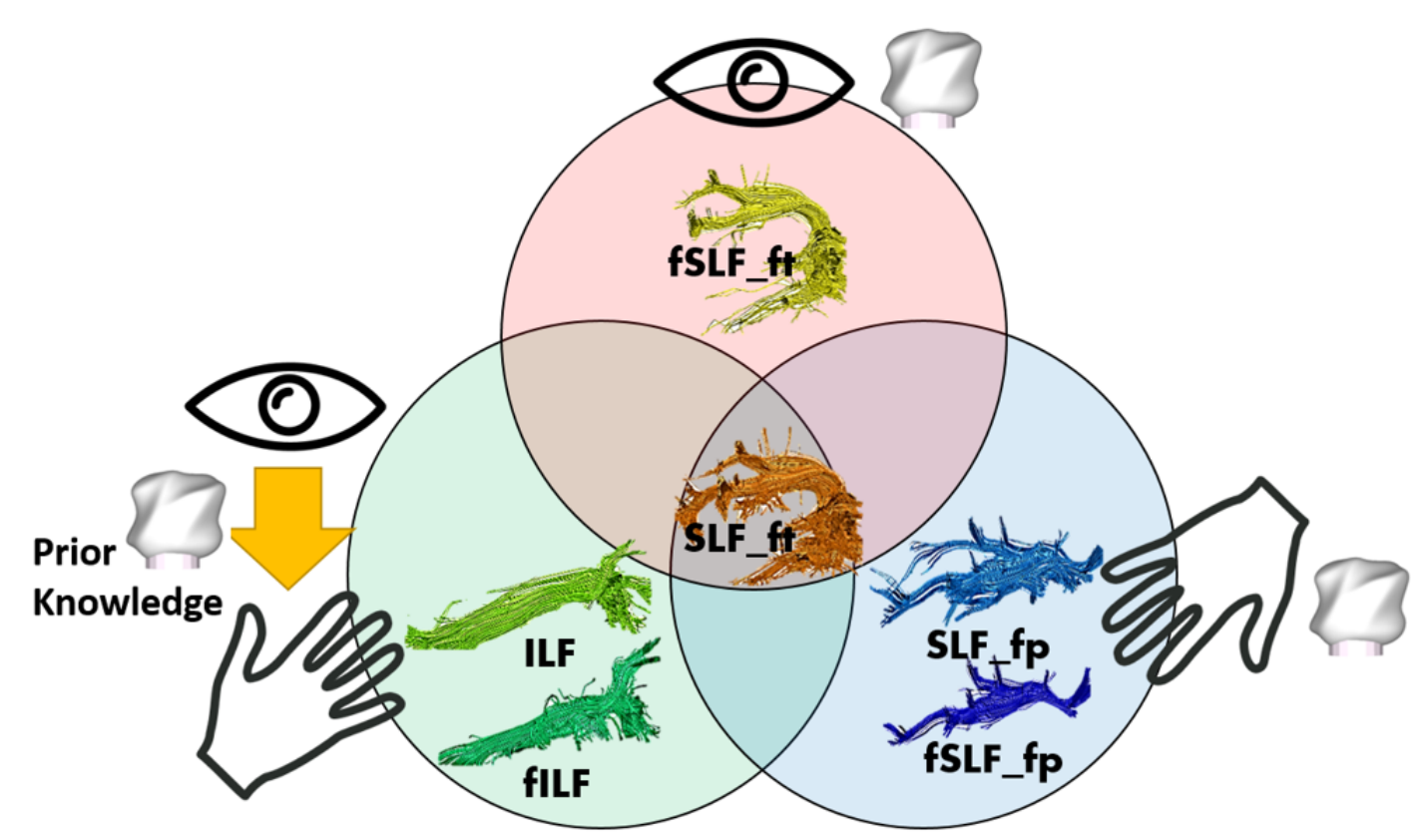

Fig. 2 This figure illustrates the WM tracks involved in multisensory shape processing: Unisensory haptic shape information is relayed by SLF_fp and fSLF_fp, whereas fSLF_ft processes unisensory visual shape information. Both ILF and fILF are involved in visuo-haptic cross-modal transfer. Finally, SLF_ft (overlapping with the arcuate fasciculus) is implicated in all aspects of multisensory processing (visual, haptic, visuo-haptic). 